\title{
Transcatheter Aortic Valve Implantation Complicated With Asymptomatic Left Ventricular Dissection and Conservative Management
}

\author{
Allende-Carrera, Ricardo; Aguilar-Rojas, Luis Antonio. \\ Cardiology department. Hospital de Especialidades Médicas de la Salud. San Luis Potosi, \\ Mexico.
}

\section{Introduction}

Within the last decade, transcatheter aortic valve implantation (TAVI) has emerged as a valuable alternative to surgical aortic valve replacement (SAVR) in an increasingly wide spectrum of patients with severe symptomatic aortic stenosis. Major complications still occur in $\leq 6 \%$ to $8 \%$ of cases. Such major complications have been associated with a 2 - to 3 -fold increase in 30-day mortality. ${ }^{1} \mathrm{We}$ describe a case of TAVI complicated with asymptomatic left ventricular dissection.

\section{Case Presentation}

We present a case of an 86-year-old female with history of a 5 year-diagnosed Hypertension and severe aortic stenosis on New York Heart Association class III. Left ventricle ejection fraction of $60 \%$ with a peak to peak gradient of $120 \mathrm{mmHg}$ and a valvular aortic area of 0.4 cm2 was documented. Aortic valve anatomy revealed a perimeter of $68 \mathrm{~mm}$, area of 360.3 $\mathrm{mm}^{2}$ and perimeter derived diameter of $21.6 \mathrm{~mm}$. The patient underwenta transcatheter aortic 
valve implantation under normal routine and international standardized technique. Using a 5 Fr Multipurpose catheter (Boston Scientific Corporation, Marlborough, Massachusetts, United States) and a $0.035^{\prime}$ hydrophilic Term (Terumo Corporation. Shybuya City, Tokyo, Japan) guide wire the valve was crossed successfully. The hydrophilic guide wire was changed to a medium size Safari guide wire (Boston Scientific Corporation, Marlborough, Massachusetts, United States) and replaced with a 5 French pigtail catheter. Having done that, a small Acurate Neo valve was placed sucessfully. (Boston Scientific Corporation, Marlborough, Massachusetts, United States) Noparavalvular leak was observed and peak to peak gradient of $9 \mathrm{mmHg}$ was registered. On the control Echcoardiogram, peak to peak of $8 \mathrm{mmHg}$ was registered but left ventricle free wall dissection was observed. (Figure 1) No segmentary movement disorders were observed, neither EKG changes. After Heart Team evaluation and due to the fact that the patient remained asymptomatic and hemodynamically stable, no further measures were taken. The patient was observed for 48 hours and discharged asymptomatic. The 30-day follow up revealed a left-ventricle aneurysm but improvement to functional class I was seen.(Figure 2) The patient nowadays (12 months post-TAVR) remains asymptomatic and on Class I NYHA. No changes on gradient, ejection fraction or paravalvular leak have been registered.

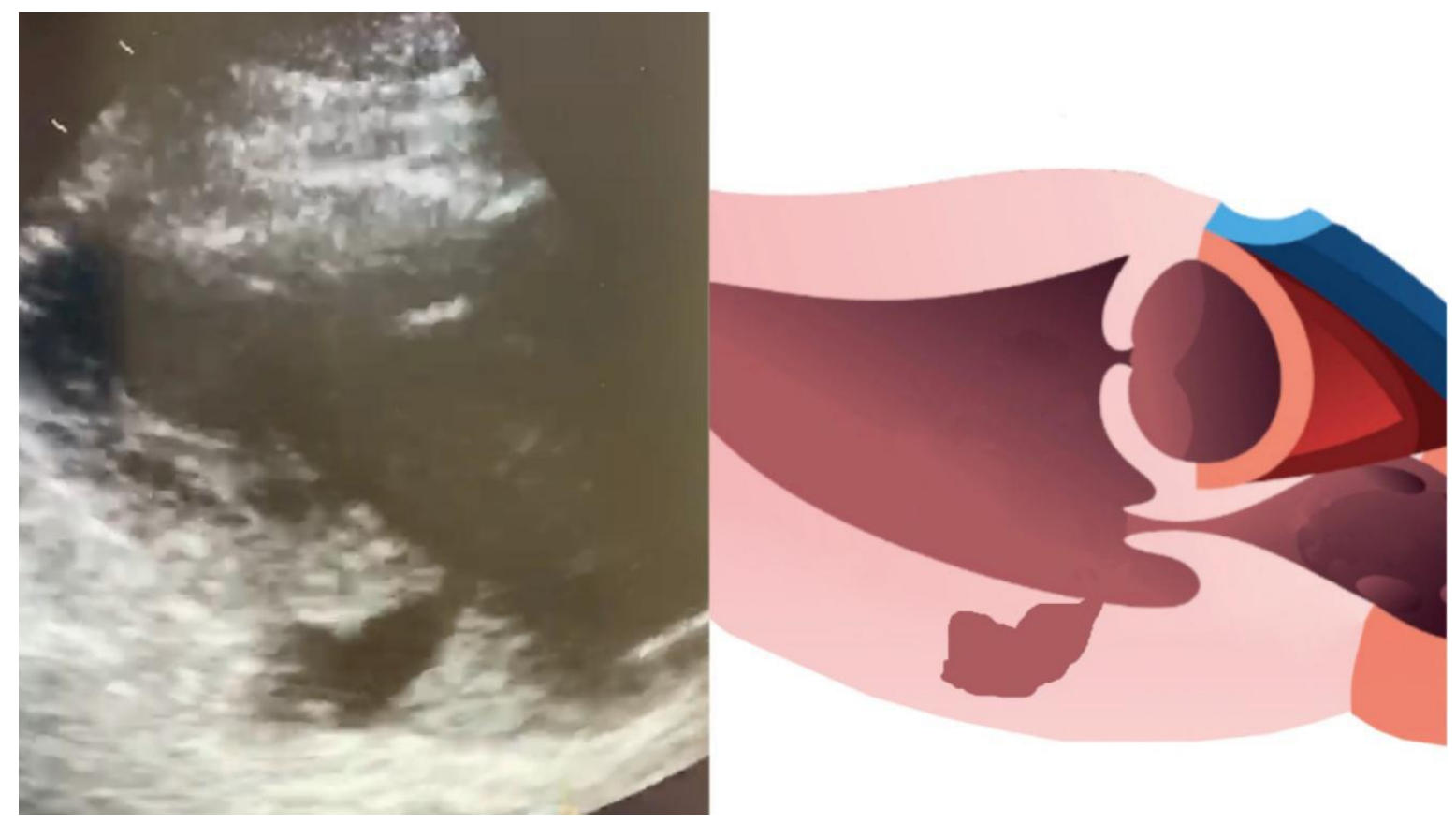

Figure 1.- (A-Left). Transprocedural echocardiogram image showing left ventricle free wall dissection with passage of blood into cavity. (B-Right). Schematic representation of left ventricle free wall dissection with passage of blood into cavity. 

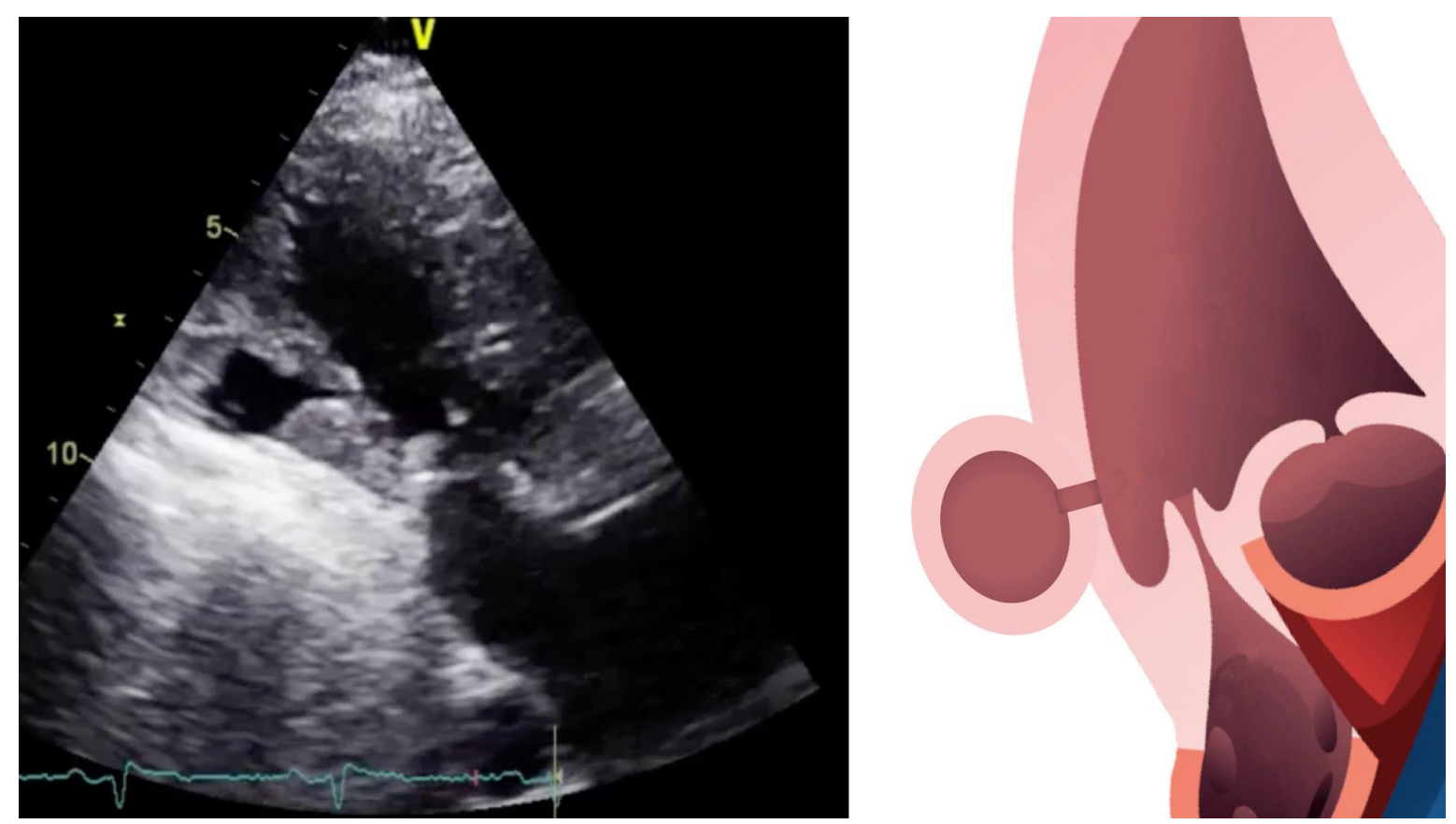

Figure 2.- (A-Left) Thirty-day echocardiogram showing the presence of a newly-developed aneurysm of the left ventricle free wall with passage of blood into cavity. (B-Right) Schematic representation of a newly-developed aneurysm of the left ventricle free wall with passage of blood into cavity.

\section{Discussion}

Intramyocardial dissection is an uncommon complication of myocardial infarction, chest trauma, and percutaneous intervention. It is usually caused by a hemorrhagic dissection among the spiral myocardial fibers after the rupture of intramyocardial vessels into the interstitial space. ${ }^{2}$ Differential diagnosis includes pseudoaneurysm following complete rupture of the myocardial wall which is sealed by the pericardium. Intramyocardial dissection is differentiated by confirming the integrity of epicardium and demonstrating partially ruptured myocardium which resulting in a cavity filled with blood. 3

The introduction of the valve device and stiff guide wires into the aortic root and left ventricle (LV) during TAVI raises the potential for injuries to the ventricle. Such injuries are infrequent, with cardiac perforation occurring in $1.3 \%$. The clinical presentation can be highly variable, ranging from rapid-onset cardiac tamponade to an indolent left ventricular pseudoaneurysm. 1 Patients who develop cardiac tamponade requiring open surgical exploration or repair have dismal outcomes, whereas outcomes are far superior in patientswho remain hemodynamically stable and can undergo conservative management or percutaneous 
repairs. 4

In a multicentre registry study comprising 27760 patients who underwent transfemoral TAVI, $212(0.76 \%)$ patients required emergency cardiac surgery, the leading cause of which was guidewire- induced LV perforation (28.3\%). ${ }^{5}$ Limiting the use of the stiffest wires and careful exchange of catheter/wires under fluoroscopy on traversing the aortic valve (ensuring that a straight wire never extends for more than a couple of $\mathrm{cm}$ into the LVOT) can reduce the risk of left ventricular perforation. ${ }^{6}$ Nielsen NE et al reported that the risk of LV perforation is eliminated by minimizing the contact time between the stiff guidewire and the LV. 7

There is a certain preference for surgical repair of the defect in LV. However, despite prompt diagnosis and treatment, mortality remains high. Pliam and Sternlieb reviewed 15 cases of intramyocardial dissecting hematoma. Out of 10 patients treated conservatively only one survived, while surgically treated patients all survived. Following this review, there are several case reports with good results and acceptable mid to long-term survival of conservatively treated patients who were hemodynamically stable. $8-9$

In our case, the procedure was carried out according to the techniques described in the international literature, always taking care of every detail to avoid catastrophic complications. However, despite all the precautions, we had ventricular dissection ascomplications. It is important to emphasize the importance of the echocardiogram in TAVI, making measurements of successful parameters, but most importantly, ruling out complications. In the described scenario described, the patient remained asymptomaticand hemodynamically stable; however, thanks to the support of the echocardiogram, a potentially catastrophic complication such as ventricular dissection was identified. Since pericardial effusion and pseudoaneurysm was ruled out, we decided to continue with expectant management, with follow-up echocardiography and close monitoring of hemodynamic parameters.

\section{Conclusion}

We describe a case of asymptomatic left ventricular dissection secondary to TAVI, a lifethreatening complication. The management of left ventricular injuries is highly specific and depends on the type of lesion, the wound location, the patient's clinical status, and the patient's overall health status and ability to tolerate an open repair. We can conclude that it is feasible to maintain a conservative and expectant management in this type of complications as 
long as pseudoaneurysm or pericardial effusion are ruled out and the patient remains hemodynamically stable.

\section{References}

[1] Langer NB, Hamid NB, Nazif TM, Khalique OK, Vahl TP, White J, Terre J, Hastings R, Leung D, Hahn RT, et al. Injuries to the aorta, aortic annulus, and left ventricle during transcatheter aortic valve replacement: management and outcomes. Circ Cardiovasc Interv. 2017;10:e004735.

[2] Pliam MB, Sternlieb JJ. Intramyocardial dissecting hematoma: An unusual form of subacute cardiac rupture. J Card Surg. 1993;8:628-37.

[3] Nagy A, Khan ZI, Al Sayari SY, Aboushaeshae AS, Natarajan R. Asymptomatic left ventricular dissection. J Cardiovasc Echography 2017;27:62-3.

[4] Agrawal Y, Kalavakunta J, Saltiel F, Gupta V. Left ventricular wall rupture and cardiac tamponade during transcatheter aortic valve replacement. Am J Respir Crit Care Med. 2016;193:A6964-A6964.

[5] Eggebrecht H, Vaquerizo B, Moris C, Bossone E, Lammer J, Czerny M et al. Incidence and outcomes of emergent cardiac surgery during trans- femoral transcatheter aortic valve implantation (TAVI): insights from the European Registry on Emergent Cardiac Surgery during TAVI (EuRECS- TAVI). Eur Heart J 2018;39:676-84.

[6] Percutaneous interventional cardiovascular medicine - The PCR-EAPCI Textbook. Transcatheter aortic valve implantation. Nicolo Piazza, Alain Cribier, Rodney De Palma.

[7] Nielsen NE, Baranowska J, Bramlage P, Baranowski J. Minimizing the risk for left ventricular rupture during transcatheter aortic valve implantation by reducing the presence of stiff guidewires in the ventricle. Interact CardioVasc Thorac Surg 2019;29:365-70.

[8] Pliam MB, Sternlieb JJ. Intramyocardial dissecting hematoma: An unusual form of subacute cardiac rupture. J Card Surg 1993;8:628-37.

[9] Galache Osuna JG, Marquina Barcos A, Cay Diarte E, Sánchez-Rubio Lezcano J, Salazar González JJ, Placer Peralta LJ. Conservative management of a post infarction intramyocardial dissecting hematoma. Rev Esp Cardiol 2003;56:735-7. 